\title{
Reliability-based Fracture Analysis for Plate Bending Problems with the Dual Boundary Element Method
}

\author{
Llewellyn Morse, ${ }^{\text {a) }}$ Zahra Sharif Khodaei, ${ }^{\text {b) }}$ and M. H. Aliabadic) \\ Department of Aeronautics, Imperial College London, South Kensington Campus, City and Guilds Building, Exhibition Road, \\ London SW7 2AZ, UK \\ a) Corresponding author: llewellyn.morse12@imperial.ac.uk \\ b) Electronic mail: z.sharif-khodaei@imperial.ac.uk

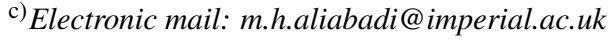

\begin{abstract}
A novel methodology is presented for the efficient reliability analysis of plate structures containing cracks with the Dual Boundary Element Method (DBEM). The derivatives of the DBEM plate formulations for the Crack Surface Displacement Extrapolation (CSDE) method have been derived for the first time and are used as part of an Implicit Differentiation Method (IDM) for the efficient calculation of Stress Intensity Factor (SIF) sensitivities. A numerical example is investigated in which results from the presented CSDE methodology are compared to those from the J-integral. The SIF sensitivities were used with the First-Order Reliability Method (FORM) to determine the reliability of a plate structure containing a crack. Results indicate that the proposed CSDE methodology is capable of providing estimates for reliability that are very similar to those provided by the J-integral. Given that the proposed CSDE methodology can be easier to implement, it could prove to be an effective alternative to the J-integral for the efficient reliability analysis of plate structures containing cracks.
\end{abstract}

\section{INTRODUCTION}

The Dual Boundary Element Method (DBEM) was developed as an effective means of modelling cracks in structures. In contrast to previous methods, mixed-mode crack problems could be solved with the DBEM in a single-region formulation; significantly reducing model complexity and the number of equations needed. Due to the effectiveness of the DBEM in fracture analysis, it has been extensively used in the research community mainly for problems involving 2D problems. Examples include topology optimisation [1], reliability analysis of structures subjected to fatigue [2], shape optimisation of hydrofoils [3], and the modelling of creep in concrete [4].

Despite its popularity for 2D problems, the DBEM has received less attention for plate problems due to the increased level of complexity; plates have more degrees of freedom (5 vs. 2), they can be subjected to the combined loading of tensile, bending, and pressure forces, and they have finite thickness along which stress can vary. Plate problems are arguably just as important to consider as 2D problems. Many engineering structures can be thought of as plates; aircraft wings and fuselages are composed of many plates joined together. The DBEM for plate problems was first developed by Dirgantara and Aliabadi [5]. This approach has since been applied to a wide range of problems by the research community, such as dynamic fracture analysis [6], the modelling of Lamb waves for Structural Health Monitoring (SHM) [7], fracture analysis with large deflection [8], and fatigue crack growth in assembled structures [9]. A comprehensive overview of the DBEM for 2D and plate problems, can be found in a book by Aliabadi [10]. Due to the widespread relevance of plates for engineering applications, they are the focus of this paper.

The calculation of the sensitivities of Stress Intensity Factors (SIFs) with respect to design variables is an important part of determining the reliability of a structure containing flaws. Among the most common methods for calculating Stress Intensity Factors (SIFs) with the DBEM is the crack Surface Displacement Extrapolation (CSDE) method and the J-integral. Since the DBEM can very accurately model displacements and stresses in the internal domain of a structure, it is very effective when used with the J-integral. However, due to its complexity it can be difficult to implement. The CSDE on the other hand is significantly easier to implement but can require the use of special quarter-point elements at crack tips to ensure sufficient accuracy. The sensitivities of SIFs with the DBEM can be calculated numerically using the relatively crude Finite Difference Method (FDM), or analytically using the more efficient Implicit Differentiation Method (IDM) which involves deriving the derivatives of the DBEM fundamental solutions. To the knowledge of the authors, there is only one example of SIF sensitivities for plates being calculated with an IDM: Morse et al. [11] derived the derivatives of the J-integral for plates to calculate SIF sensitivities for a plate with a centre crack subjected to complex loading conditions. Results were found to compare well with the FDM and the IDM was found to be significantly more efficient than the FDM. Although previous work has involved the derivatives of the J-integral being used to calculate SIF sensitivities for plates, the derivatives of the CSDE method have yet to be used for this purpose. Due to the fact that the CSDE method is significantly easier to implement it could 
prove to be an effective alternative to the J-integral for the calculation SIF sensitivities for plate structures.

In summary, this paper presents a novel methodology for the calculation of SIF sensitivities for plates with the CSDE method. The derivatives of the DBEM plate formulations for the CSDE method are derived for the first time and are used as part of an IDM for the calculation of SIF sensitivities. The methodology behind the CSDE method will first be introduced, followed by the newly derived IDM formulations. A numerical example will then be presented featuring reliability analyses on a square plate subjected to complex loading conditions that required the use of the SIF sensitivities. The accuracy of the CSDE method will then be compared to J-integral.

\section{METHODOLOGY}

\section{DBEM-based IDM for Plate Bending Problems}

For conciseness, the DBEM integral equations and their derivatives for plate bending are omitted. The DBEM integral equations for plate bending can be found in [10], while their derivatives with respect to some geometric parameter $Z_{m}$ can be found in [11].

The IDM involves taking the derivative of the system of equations from DBEM with respect to some geometric parameter $Z_{m}$ (e.g. plate length). The derivative of the system of equations is:

$$
\mathbf{A} \mathbf{X}_{, m}=\left[\mathbf{F}_{, m}-\mathbf{A}_{, m} \mathbf{X}\right]
$$

where $\mathbf{A}$ and $\mathbf{X}$ can be obtained from DBEM. Because the right-hand side of equation (1) is known, LU decomposition can be used to obtain the unknown derivatives of boundary displacements and tractions $\mathbf{X}_{, m}$.

The calculation time of the matrix $\mathbf{A}_{, m}$ is significantly less than might be expected since when both the collocation node and the field point lie on a fixed boundary that is unaffected by a change in the geometric parameter $Z_{m}$, the corresponding elements in $\mathbf{A}_{m}$ are zero. This has the effect of significantly improving the efficiency of the proposed methodology.

\section{Stress Intensity Factor Evaluation}

The CSDE method is used in this work for the calculation of SIFS and their sensitivities. It involves the linear extrapolation of the SIFs near the crack tip. A DBEM mesh of a crack tip with discontinuous quadratic elements can be seen in Figure 1. Some of the nodes near the crack tip, e.g. nodes A, B, C, and D, are labelled.

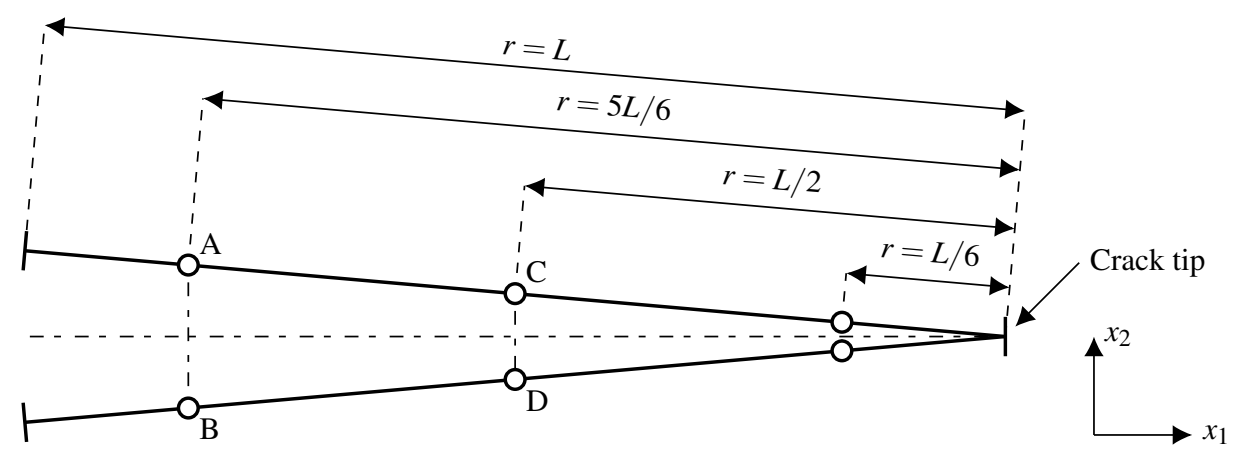

FIGURE 1: Nodes near a crack tip. The global coordinate system is shown.

The SIFs can be calculated at a certain distance $r$ from the crack tip shown in Figure 1 using the following equations:

$$
K_{I}^{m}(r)=\frac{E h \sqrt{2 \pi}}{8 \sqrt{r}}\left|\Delta u_{2}(r)\right| \quad K_{I I}^{m}(r)=\frac{E h \sqrt{2 \pi}}{8 \sqrt{r}}\left|\Delta u_{1}(r)\right|
$$




$$
K_{I}^{b}(r)=\frac{E h^{3} \sqrt{\pi / 2}}{48 \sqrt{r}}\left|\Delta w_{2}(r)\right| \quad K_{I I}^{b}(r)=\frac{E h^{3} \sqrt{\pi / 2}}{48 \sqrt{r}}\left|\Delta w_{1}(r)\right| \quad K_{I I I}^{b}(r)=\frac{5 E h \sqrt{\pi / 2}}{24(1+v) \sqrt{r}}\left|\Delta w_{3}(r)\right|
$$

where the superscripts $m$ and $b$ denote membrane and bending SIFs respectively. $w_{1}$ and $w_{2}$ denote rotations in the directions $x_{1}$ and $x_{2}$ respectively, and $w_{3}$ denotes displacement in the direction $x_{3}$ (out-of-plane). $u_{1}$ and $u_{2}$ are the displacements in the directions $x_{1}$ and $x_{2}$ respectively. $\Delta$ indicates the difference across the crack tip, e.g. $\left|\Delta u_{1}(r=L / 2)\right|=\left|u_{1}^{C}-u_{1}^{D}\right|$, where $u_{1}^{C}$ and $u_{1}^{D}$ are the values of $u_{1}$ evaluated at nodes $\mathrm{C}$ and $\mathrm{D}$ respectively in Figure 1.

By conducting linear extrapolation using equations (2) and (3), the SIFs at the crack tip can be obtained:

$$
K_{\alpha}^{m}=R_{C D} K_{\alpha}^{m, C D}-R_{A B} K_{\alpha}^{m, A B} \quad K_{i}^{b}=R_{C D} K_{i}^{b, C D}-R_{A B} K_{i}^{b, A B}
$$

where the subscript $\alpha$ can take values of $I$ or $I I$, and the subscript $i$ can take values of $I, I I$, or $I I I . R_{A B}$ and $R_{C D}$ are defined as: $R_{A B}=r_{C D} /\left(r_{A B}-r_{C D}\right)$ and $R_{C D}=r_{A B} /\left(r_{A B}-r_{C D}\right)$, where $r_{A B}$ and $r_{C D}$ are the distances of the nodes on the lines $\mathrm{AB}$ and $\mathrm{CD}$ respectively to the crack tip. The maximum values of the SIFs through the thickness of the plate are:

$$
K_{I}^{\max }=\frac{K_{I}^{m}}{h}+\frac{6}{h^{2}} K_{I}^{b} \quad K_{I I}^{\max }=\frac{K_{I I}^{m}}{h}+\frac{6}{h^{2}} K_{I I}^{b} \quad K_{I I I}^{\max }=\frac{3}{2 h} K_{I I I}^{b}
$$

\section{Stress Intensity Factor Sensitivity Evaluation}

By taking the derivatives of equations (2) and (3) with respect to some geometric parameter $Z_{m}$, the derivatives of the SIFs can be calculated at a certain distance $r$ from the crack tip:

$$
\begin{gathered}
K_{I, m}^{m}(r)=\frac{E h \sqrt{2 \pi}}{8 \sqrt{r}}\left[\frac{\Delta u_{2}(r)}{\left|\Delta u_{2}(r)\right|} \Delta u_{2, m}(r)-\frac{r_{, m}}{2 r}\left|\Delta u_{2}(r)\right|\right] \\
K_{I I, m}^{m}(r)=\frac{E h \sqrt{2 \pi}}{8 \sqrt{r}}\left[\frac{\Delta u_{1}(r)}{\left|\Delta u_{1}(r)\right|} \Delta u_{1, m}(r)-\frac{r_{, m}}{2 r}\left|\Delta u_{1}(r)\right|\right] \\
K_{I, m}^{b}(r)=\frac{E h^{3} \sqrt{\pi / 2}}{48 \sqrt{r}}\left[\frac{\Delta w_{2}(r)}{\left|\Delta w_{2}(r)\right|} \Delta w_{2, m}(r)-\frac{r_{, m}}{2 r}\left|\Delta w_{2}(r)\right|\right] \\
K_{I I, m}^{b}(r)=\frac{E h^{3} \sqrt{\pi / 2}}{48 \sqrt{r}}\left[\frac{\Delta w_{1}(r)}{\left|\Delta w_{1}(r)\right|} \Delta w_{1, m}(r)-\frac{r_{, m}}{2 r}\left|\Delta w_{1}(r)\right|\right] \\
K_{I I I, m}^{b}(r)=\frac{5 E h \sqrt{\pi / 2}}{24(1+v) \sqrt{r}}\left[\frac{\Delta w_{3}(r)}{\left|\Delta w_{3}(r)\right|} \Delta w_{3, m}(r)-\frac{r_{, m}}{2 r}\left|\Delta w_{3}(r)\right|\right]
\end{gathered}
$$

By conducting linear extrapolation using equations (6-10), the sensitivities of the SIFs at the crack tip can be obtained:

$$
\begin{gathered}
K_{\alpha, m}^{m}=\left(R_{C D, m} K_{\alpha}^{m, C D}+R_{C D} K_{\alpha, m}^{m, C D}\right)-\left(R_{A B, m} K_{\alpha}^{m, A B}+R_{A B} K_{\alpha, m}^{m, A B}\right) \\
K_{i, m}^{b}=\left(R_{C D, m} K_{i}^{b, C D}+R_{C D} K_{i, m}^{b, C D}\right)-\left(R_{A B, m} K_{i}^{b, A B}+R_{A B} K_{i, m}^{b, A B}\right)
\end{gathered}
$$

where the subscript $\alpha$ can take values of $I$ or $I I$, and the subscript $i$ can take values of $I, I I$, or $I I I . R_{A B, m}$ and $R_{C D, m}$ are defined as: $R_{A B, m}=\left[r_{C D, m}-R_{A B}\left(r_{A B, m}-r_{C D, m}\right)\right] /\left(r_{A B}-r_{C D}\right)$ and $R_{C D, m}=\left[r_{A B, m}-R_{C D}\left(r_{A B, m}-r_{C D, m}\right)\right] /\left(r_{A B}-r_{C D}\right)$, where $r_{A B, m}$ and $r_{C D, m}$ are the derivatives of the distances of the nodes on the lines $\mathrm{AB}$ and $\mathrm{CD}$ respectively to the crack tip. By taking the derivatives of equation (5), the sensitivities of the maximum values of the SIFs through the thickness of the plate are:

$$
K_{I, m}^{\max }=\frac{K_{I, m}^{m}}{h}+\frac{6}{h^{2}} K_{I, m}^{b} \quad K_{I I, m}^{\max }=\frac{K_{I I, m}^{m}}{h}+\frac{6}{h^{2}} K_{I I, m}^{b} \quad K_{I I I, m}^{\max }=\frac{3}{2 h} K_{I I I, m}^{b}
$$




\section{NUMERICAL EXAMPLE}

To test the accuracy of the proposed CSDE methodology, a numerical example featuring a square steel plate with a crack was investigated and results were compared to the J-integral method. The plate can be seen in Figure 2, and a zoomed-in view of the crack at the top-left hole can be seen in Figure 3. The plate has straight edges and curved corners. Through-thickness holes are present at the corners. The plate is subjected to uniform pressure over its entire upper surface and combined biaxial tension and bending on the straight edges. The plate is simply supported such that the straight outer edges cannot displace in the $x_{3}$ direction. Reliability analyses were conducted on this plate using the 16 random variables seen in Table I. The First-Order Reliability Method (FORM) was used to calculate reliability indices of the plate for a range of Coefficients of Variation $(\mathrm{CoV})$ of the crack length $a$ between 0.05 and 0.5 . A good introduction to the FORM and other reliability methods is [12].

A DBEM mesh consisting of 132 quadratic elements was used. The four straight outer edges were composed of 12 elements each. The four outer curved corners were composed of 3 elements each. The top-left hole was composed of 30 elements, and the other three holes were composed of 4 elements each. The crack originating from the upper-left hole was composed of 15 elements on each surface. This DBEM mesh was found to provide sufficient converge within $1 \%$ when calculating the stress intensity factors at the crack tip with both the CSDE method and the J-integral.

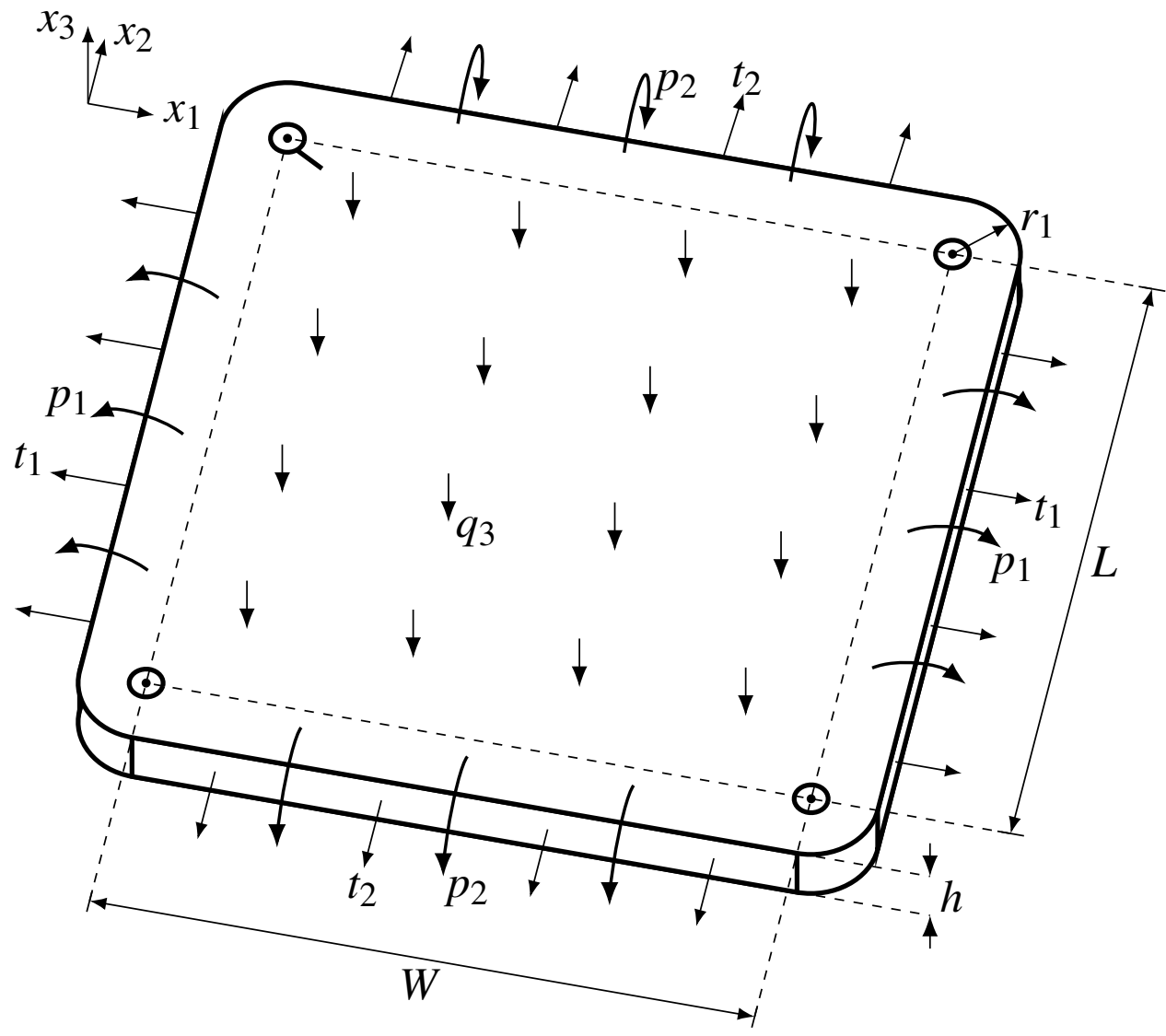

FIGURE 2: Square steel plate with a crack subjected to uniform pressure and biaxial tension and bending. The crack can be seen originating from the top-left hole. 


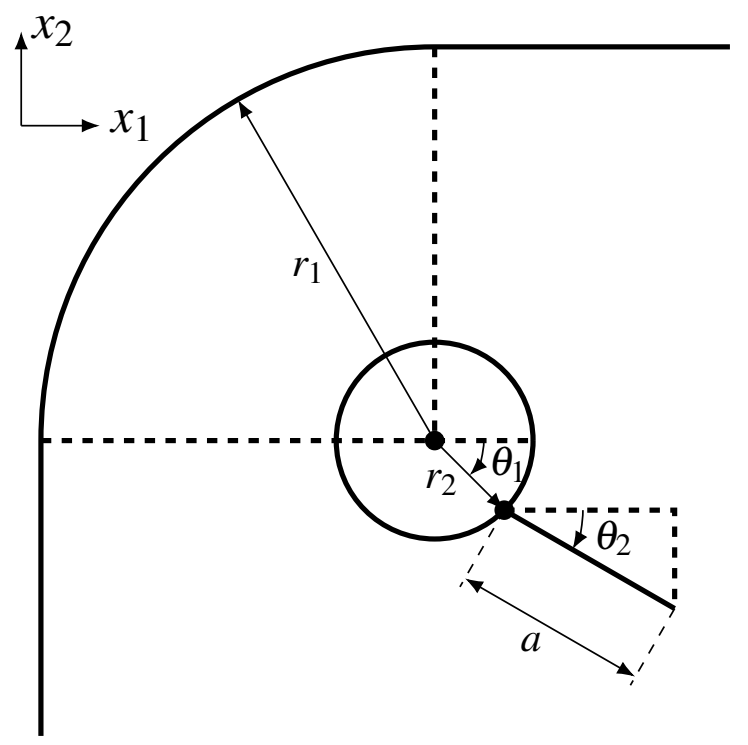

FIGURE 3: A zoomed-in view of the crack originating from the top-left hole.

TABLE I: The 16 random variables and their probability distributions.

\begin{tabular}{|c|c|c|c|c|c|c|}
\hline$Z_{i}$ & $X_{i}$ & Parameter & Description & Distribution & Mean & $\mathrm{CoV}$ \\
\hline$\overline{Z_{1}}$ & $X_{1}$ & $L$ & Plate length & Normal & $1 \mathrm{~m}$ & 0.05 \\
\hline$Z_{2}$ & $X_{2}$ & $W$ & Plate width & Lognormal & $1 \mathrm{~m}$ & 0.05 \\
\hline$Z_{3}$ & $X_{3}$ & $r_{1}$ & Corner radius & Lognormal & $0.1 \mathrm{~m}$ & 0.05 \\
\hline$Z_{4}$ & $X_{4}$ & $r_{2}$ & Hole radius & Normal & $0.025 \mathrm{~m}$ & 0.05 \\
\hline$Z_{5}$ & $X_{5}$ & $\theta_{1}$ & Angle to crack & Lognormal & $45^{\circ}$ & 0.05 \\
\hline$Z_{6}$ & $X_{6}$ & $\theta_{2}$ & Angle of crack & Normal & $30^{\circ}$ & 0.05 \\
\hline$Z_{7}$ & $X_{7}$ & $a^{2}$ & Crack length & Lognormal & $0.05 \mathrm{~m}$ & Variable \\
\hline$Z_{8}$ & $X_{8}$ & $h$ & Plate thickness & Normal & $0.1 \mathrm{~m}$ & 0.05 \\
\hline$Z_{9}$ & $X_{9}$ & $t_{1}$ & Membrane traction in direction $x_{1}$ & Lognormal & $8 \mathrm{MN} / \mathrm{m}$ & 0.10 \\
\hline$Z_{10}$ & $X_{10}$ & $t_{2}$ & Membrane traction in direction $x_{2}$ & Normal & $9 \mathrm{MN} / \mathrm{m}$ & 0.10 \\
\hline$Z_{11}$ & $X_{11}$ & $p_{1}$ & Bending moment in direction $x_{1}$ & Lognormal & $1 \mathrm{MN}$ & 0.10 \\
\hline$Z_{12}$ & $X_{12}$ & $p_{2}$ & Bending moment in direction $x_{2}$ & Lognormal & $1.5 \mathrm{MN}$ & 0.10 \\
\hline$Z_{13}$ & $X_{13}$ & $q_{3}$ & Uniform pressure & Normal & $1 \mathrm{MN} / \mathrm{m}^{2}$ & 0.10 \\
\hline$Z_{14}$ & $X_{14}$ & $E$ & Young's modulus & Lognormal & $206.8 \mathrm{GPa}$ & 0.20 \\
\hline$Z_{15}$ & $X_{15}$ & $v$ & Poisson's ratio & Lognormal & 0.29 & 0.20 \\
\hline$Z_{16}$ & - & $G_{o}$ & Strain energy release rate required for crack growth onset & Lognormal & $200 \mathrm{~J} / \mathrm{m}^{2}$ & 0.20 \\
\hline
\end{tabular}

The failure condition of this plate is with regards to the onset of crack growth. The Limit State Function (LSF) $g$ in this case is:

$$
g(\mathbf{Z})=G_{o}-G_{e f f}(\mathbf{X})
$$

where $G_{o}$ is the strain energy release rate required for the onset of crack growth, and $G_{e f f}$ is the effective strain energy release rate at the crack tip. $\mathbf{Z}$ is composed of the random variables that influence $g: \mathbf{Z}=\left(L, W, r_{1}, r_{2}, \theta_{1}, \theta_{2}, a, h, t_{1}\right.$, $\left.t_{2}, p_{1}, p_{2}, q_{3}, E, v, G_{o}\right)$, while the vector $\mathbf{X}$ contains the random variables that influence $G_{e f f}: \mathbf{X}=\left(L, W, r_{1}, r_{2}, \theta_{1}, \theta_{2}\right.$, $\left.a, h, t_{1}, t_{2}, p_{1}, p_{2}, q_{3}, E, v, G_{o}\right)$. Ideally, $G_{o}$ should be found experimentally. However, for demonstration purposes, $G_{o}$ is chosen arbitrarily.

Dirgantara and Aliabadi [13] proposed an equation for $G_{e f f}$ :

$$
G_{e f f}(\mathbf{X})=G_{I}^{m}(\mathbf{X})+T_{I} G_{I}^{b}(\mathbf{X})+T_{I I}\left(G_{I I}^{m}(\mathbf{X})+G_{I I}^{b}(\mathbf{X})+G_{I I I}^{b}(\mathbf{X})\right), 0 \leq T_{I} \leq 1,0 \leq T_{I I} \leq 1
$$


where $T_{I}$ and $T_{I I}$ are coefficients ideally found from experiments. However, in this work they were both arbitraily chosen to be 0.5 . The individual components e.g. $G_{I}^{m}, G_{I}^{b}$, etc. are calculated using the individual stress intensity factors e.g. $K_{I}^{m}, K_{I}^{b}$, etc. Their expressions can be found in [13]. The sensitivity of $G_{e f f}$ with respect to some geometric variable $Z_{m}$ is:

$$
G_{e f f, m}(\mathbf{X})=G_{I, m}^{m}(\mathbf{X})+T_{I} G_{I, m}^{b}(\mathbf{X})+T_{I I}\left(G_{I I, m}^{m}(\mathbf{X})+G_{I I, m}^{b}(\mathbf{X})+G_{I I I, m}^{b}(\mathbf{X})\right), 0 \leq T_{I} \leq 1,0 \leq T_{I I} \leq 1
$$

By using equations (15) and (16) with the LSF seen in equation (14), the FORM can be used to obtain estimates for the reliability of the structure with regards to the onset of crack growth. The results of this can be seen in Figure 4 for a range of $\mathrm{CoV}$ values for $a\left(\mathrm{CoV}_{a}\right)$ from 0.05 to 0.5 . The left axis presents the reliability index $\beta$, and the right axis presents the probability of failure $P_{F}$. The relationship between these two parameters is:

$$
P_{R}=1-P_{F}=1-\Phi(-\beta)=\Phi(\beta)
$$

where $\Phi$ is the CDF of the standard normal distribution.

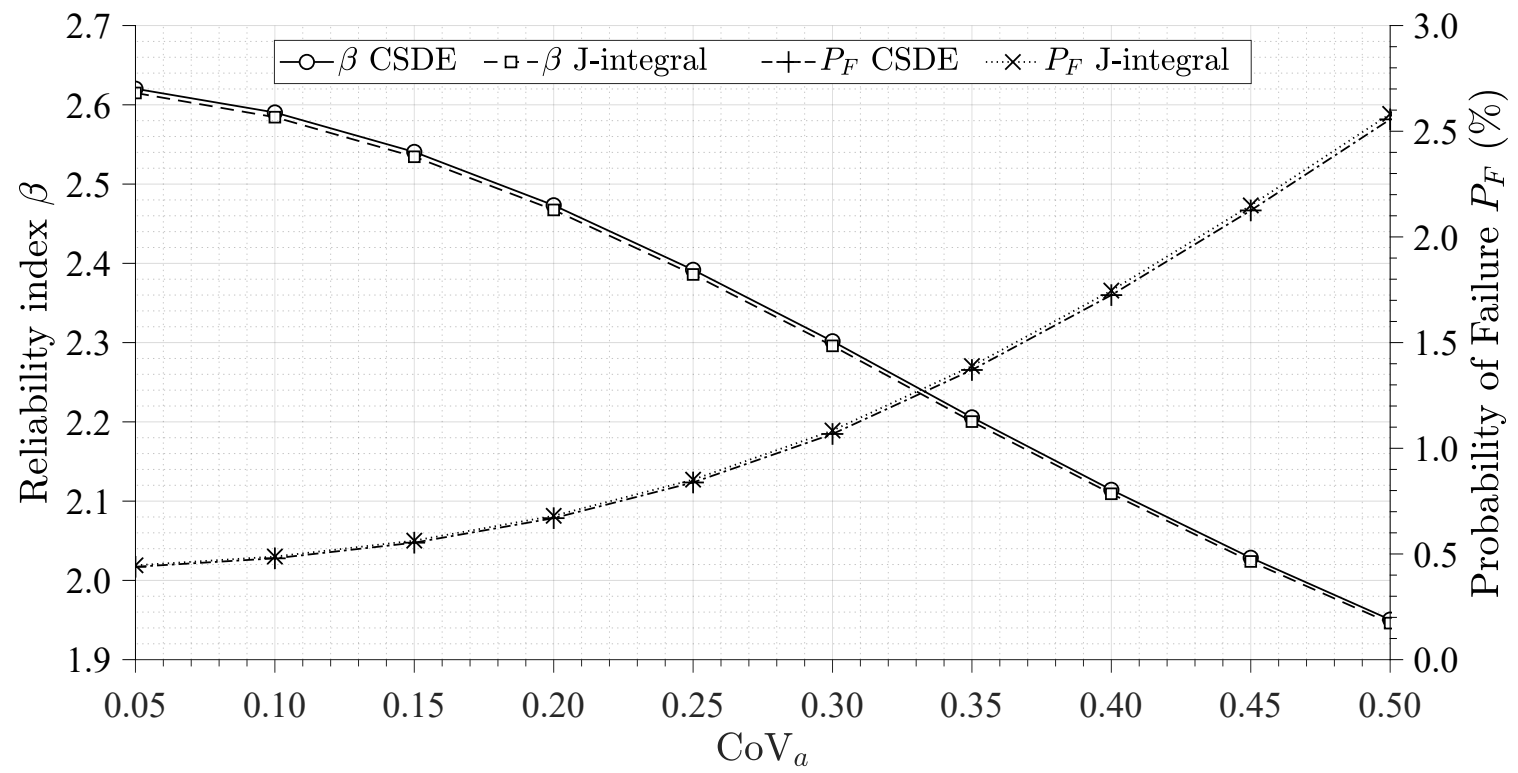

FIGURE 4: Reliability indices and probabilities of failure calculated using the FORM with the CSDE method and the J-integral.

It can be seen in Figure 4 that, as expected, the reliability index decreases and $P_{F}$ increases as $\operatorname{CoV}_{a}$ increases. Furthermore, the CSDE and the J-integral compare very well for each value of $\mathrm{CoV}_{a}$. The average difference in $\beta$ between the two methods was less than $1 \%$ at $0.23 \%$. 


\section{CONCLUSION}

In conclusion, a novel methodology was presented for the calculation of Stress Intensity Factor (SIF) sensitivities with the Crack Surface Displacement Extrapolation (CSDE) method. A numerical example featuring a square steel plate with a crack subjected to complex loading conditions was investigated. Reliability analyses were conducted on this plate with the First-Order Reliability Method (FORM) using the SIF sensitivities from the CSDE method and the J-integral. Results suggest that the CSDE and the J-integral compare well and the average difference between the two methods when calculating reliability indices was only $0.23 \%$. These results indicate that the proposed CSDE methodology possesses a similar level of accuracy to the J-integral for the calculation of SIF sensitivities. Given that the proposed CSDE methodology is easier to implement, it could prove to be an effective alternative to the J-integral for the calculation of the SIF sensitivities for plate bending problems.

\section{ACKNOWLEDGMENTS}

This research was supported by a grant provided by the Engineering and Physical Sciences Research Council (EPSRC).

\section{REFERENCES}

1. H. L. Oliveira, A. Chateauneuf, and E. D. Leonel, "Boundary element method applied to decision-making problems involving geometric variabilities in topology optimization,” Engineering Analysis with Boundary Elements 85, 116-126 (2017).

2. H. L. Oliveira, A. Chateauneuf, and W. S. Venturini, "Probabilistic crack growth analyses using a boundary element model: Applications in linear elastic fracture and fatigue problems," Engineering Analysis with Boundary Elements 36, 944-959 (2012).

3. K. V. Kostas, A. I. Ginnis, C. G. Politis, and P. D. Kaklis, "Shape-optimization of 2D hydrofoils using an isogeometric BEM solver," Computer-Aided Design 82, 79-87 (2017).

4. H. L. Oliveira, A. Chateauneuf, and E. D. Leonel, "Probabilistic mechanical modelling of concrete creep based on the boundary element method," Advances in Structural Engineering 22, 337-348 (2018).

5. T. Dirgantara, Boundary element analysis of cracks in shear deformable plates and shells, Ph.D. thesis, Department of Engineering, Queen Mary, University of London (2000).

6. J. Li, Z. Sharif Khodaei, and M. H. Aliabadi, "Dynamic fracture analysis of Kane-Mindlin plates using the dual boundary element method," Engineering Analysis with Boundary Elements 106, 217-227 (2019).

7. J. Li, Z. Sharif Khodaei, and M. H. Aliabadi, "Modelling of the high-frequency fundamental symmetric lamb wave using a new boundary element formulation," International Journal of Mechanical Sciences 155, 235-247 (2019).

8. C. Di Pisa, M. H. Aliabadi, and A. Young, "Dual boundary method for assembled plate structures undergoing large deflection," International Journal for Numerical Methods in Engineering 89, 1720-1738 (2012).

9. C. Di Pisa and M. H. Aliabadi, "Fatigue crack growth analysis of assembled plate structures with dual boundary element method," Engineering Fracture Mechanics 98, 200-213 (2013).

10. M. H. Aliabadi, The Boundary Element Method: Applications in solids and structures, Vol. 2 (John Wiley and Sons, 2002).

11. L. Morse, Z. Sharif Khodaei, and M. H. Aliabadi, "A Dual Boundary Element based Implicit Differentiation Method for Determining Stress Intensity Factor Sensitivities for Plate Bending Problems," Engineering Analysis with Boundary Elements 106, 412-426 (2019).

12. A. Nowak and K. Collins, Reliability of Structures (Boca Raton : CRC Press, 2013).

13. T. Dirgantara and M. H. Aliabadi, "Numerical simulation of fatigue crack growth in pressurized shells," International Journal of Fatigue 24, 725-738 (2002). 